\title{
RESULTS OF FINAL FOCUS TEST BEAM*
}

\author{
V. A. Alexandrof, V. Balakin, A. Mikhailichenko, \\ Budker Institute of Nuclear Physics, Novosibirsk and Protvino, Russia \\ K. Fld̀̀tmann, F. Peters, G.-A. Voss, \\ Deutsches Elektronen Synchrotron, Hamburg, Germany \\ V. Bharadwaj, M. Halling, J. A. Holt, \\ Fermi National Accelerator Laboratory, Batavia, Illinois, U.S.A. \\ J. Buon, J. Jeanjean, F. LeDiberder, V. Lepeltier, P. Puzo, \\ Laboratoire de L'Acc Âlêtrateur Linềire, Orsay, France \\ G. Heimlinger, R. Settles, U. Stierlin, \\ Max-Planck-Institut fì্r Physik, Werner-Heisenberg Institute, Munich, Germany \\ N. Akasaka, H. Hayano, N. Ishihara, H. Nakayama, K. Oide, T. Shintake, Y. Takeuchi, N. Yamamoto, \\ National Laboratory for High Energy Physics, Tsukuba, Japan \\ F. Bulos, D. Burke, R. Field, S. Hartman, R. Helm, J. Irwin, R. Iverson, P. Raimondi, S. Rokni, G. Roy, W. \\ Spence, P. Tenenbaum, S. R. Wagner, D. Walz, S. Williams, \\ Stanford Linear Accelerator Center, Stanford, California, U.S.A.
}

\begin{abstract}
The beam experiments of Final Focus Test Beam(FFTB) started in September 1993 at SLAC, and have produced a $1.7 \mu \mathrm{m} \times$ $75 \mathrm{~nm}$ spot of $46 \mathrm{GeV}$ electron beam. A number of new techniques involving two nanometer spot-size monitors have been developed. Several beam diagnostic/tuning schemes are applied to achieve and maintain the small spot. This experiment opens the way toward the nanometer world for future linear colliders.
\end{abstract}

\section{GOAL OF FFTB}

A small spot size is the most important key to enhance the luminosity of an $\mathrm{e}^{+} \mathrm{e}^{-}$linear collider. Up to now, most plans of future linear colliders at $0.5 \pm 1 \mathrm{TeV}$ center of mass assume a vertical spot size below $10 \mathrm{~nm}$ either as the standard or upgraded design number. A very ${ }^{-}$at beam with an aspect ratio more than 100 will be favored to reduce various beam-beam effects at the collision while keeping the luminosity high. The goal of FFTB[1] is to squeeze a high-energy electron beam down to $1 \mu \mathrm{m} \times 60 \mathrm{~nm}$, which is a suitable intermediate step to the future machines. FFTB is located at the end of the SLC Linac on the straight line extended from the SLAC Linac, beyond the beam switch yard. The length of the beam line is about $360 \mathrm{~m}$ from the switch yard to the beam dump, and the ${ }^{\circledR}$ nal focus section with chromaticity correction is $185 \mathrm{~m}$ long.

A small spot requires two basic factors \pm a small emittance and strong focusing (small $\beta$-functions). FFTB uses the $46 \mathrm{GeV}$ beam, accelerated by the SLAC Linac, with invariant emittances of $\gamma \varepsilon_{x} \times \gamma \varepsilon_{y}=30 \times 3 \mu \mathrm{m}^{2}$, which are the smallest among the existing high energy electron beams. The $\beta$-functions at the focal point(FP) of FFTB are $\beta_{x}^{*}=3 \mathrm{~mm}$ and $\beta_{y}^{*}=100 \mu \mathrm{m}$, which are equal to future machines. Such small beta functions inevitably induce high chromaticities in the ${ }^{\circledR}$ nal lenses. If no chromaticity correction is applied, the spot at the FP becomes 23 times

\footnotetext{
*Work supported by the Department of Energy, contract $\mathrm{DE} \pm \mathrm{AC} 03 \pm 76 \mathrm{SF} 00515$.
}

bigger in horizontal and 34 times in vertical for the design momentum spread of $\Delta p / p= \pm 0.4 \%$. Thus special focusing optics with a powerful chromatic correction system are required[2]. The chromaticity correction of FFTB is done by two-family noninterleaved sextupoles, which has become the common base design of future ${ }^{\circledR}$ nal focus systems. This con®guration suppresses the chromatic aberration as shown in Fig. 1, while canceling the major geometric aberrations of the sextupoles with the $-I$ transformer between them. The system minimizes the residual aberrations caused by the ${ }^{\circledR}$ nite thickness of the sextupoles and the optical mismatch due to the synchrotron radiation in the dipole magnets between the sextupoles and the ${ }^{\circledR}$ nal lenses.

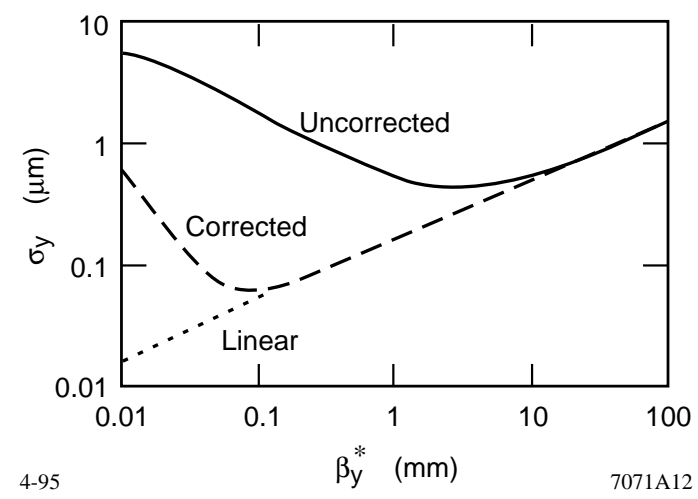

Figure. 1. The vertical spot size of FFTB as a function of the $\beta_{y}^{*}$. The dashed/solid lines are with/without the chromaticity correction. At the design value $\beta_{y}^{*}=0.1 \mathrm{~mm}$, the spot size becomes nearly the minimum, since below that point, residual aberrations start to dominate.

\section{NEW INSTRUMENTATIONS}

To achieve the design spot of FFTB, many new instrumentations have been developed and applied. Since most of their fea- 
tures will also be necessary for future machines, the development of these devices is an important part of the FFTB project.

\section{A. Magnets and power supplies}

Since the $\beta$-function peaks at the $®$ nal three quadrupoles $\left(\beta_{x}=26000 \mathrm{~m}, \beta_{y}=11000 \mathrm{~m}\right)$, the nonlinear components of these magnets should be minimized. The fabrication accuracy of the pole-faces of these magnets are made better than $\pm 2 \mu \mathrm{m}[3]$. One of the $®$ nal three quadrupoles has a pole-tip made of permendur to achieve the ®eld of $1.4 \mathrm{~T}$. The stability criteria of magnet strength in FFTB is typically $10^{-5}$ for the dipoles and a few quadrupoles[4]. Thus the power supplies of those magnets are carefully chosen to satisfy the condition. The air in the tunnel of FFTB is simply sealed without air-conditioning. Since the power consumption in the tunnel is small and also constant, this is a good choice to avoid an arti®cial temperature change due to the air-conditioning itself.

\section{B. Magnet supports}

Quadrupoles except the $®$ nal three lenses are mounted on a magnet mover supported by a pillar made of Anocast arti®cial stone. This setting satis®es the long-term stability criteria of the vertical motion of each quadrupole, $\Delta y \sim 2 \mu \mathrm{m}$ under the expected temperature change of the tunnel. The heavy $®$ nal three lenses are supported by a common table with a precision mover[5]. The Laser-Compton monitor is attached to the table to reduce the effect from the possible vibration.

\section{Initial alignment}

The initial alignment of each component is done in two steps[6]. First, alignment of several ${ }^{a}$ hinge points ${ }^{\circ}$ is done using the alignment laser extended from Linac as the reference. Second, each magnet is aligned from these hinge points by a lasertracker. The over-all accuracy of the initial transverse alignment is about $200 \mu \mathrm{m}$ through the FFTB beam line, which is within the adjustable range of the magnet movers by the beam-based alignment.

\section{Wire alignment system}

The wire alignment system is a unique tool in FFTB to detect the transverse drift of each magnet. All quadrupoles and sextupoles have three sensors attached on two invar plates sit on the median split plane (one on one end, two on the other). The sensor detects the rf signal applied on the wire like a BPM, and the resolution of the transverse wire position is $100 \mathrm{~nm}$ with a dynamic range of $\pm 1.5 \mathrm{~mm}$. A pair of wires are stretched over quasistraight sections of FFTB, and the three sensors measure the $x$ and $y$ displacements and the rolls of each magnet. The stability of the motion of the magnets detected by the system was typically less than $\pm 5 \mu \mathrm{m}$ in the upstream end of the beam line, and $\pm 15 \mu \mathrm{m}$ in the downstream end, per 24 hours. Besides a periodic day-night variation, a slow drift of $5 \mu \mathrm{m}$ /day is observed. The drift in the electronics and the wire itself were negligibly small.

\section{E. Magnet movers}

The magnet mover for the standard quadrupoles and sextupoles is so designed as to match the requirement of the tuning of the FFTB optics[7]. It is used not only in the beam-based alignment, but also in the lattice diagnostics to create bump orbits, and in the tuning process of the spot size as the multi knob with the sextupoles. This mover consists of three cam shafts independently driven by stepping motors, and the magnet sits on them with wedges. By the combination of the rotation angles of these three cams, the transverse displacement and the roll in $x$ $y$ plane are controlled independently. The resolution of the motion is $1 / 3 \mu \mathrm{m}$ and $1 \mu \mathrm{rad}$. The movable range is $\pm 1.8 \mathrm{~mm}$ in $x, \pm 1.5 \mathrm{~mm}$ in $y$, and $\pm 5 \mathrm{mrad}$ in roll. The motion is also monitored by linear gauges, and there is neither backlash nor hysteresis.

\section{F. Beam position monitor}

The beam position monitor(BPM) of FFTB is designed to detect pulse-to-pulse $x$ - and $y$-position with a resolution of $1 \mu \mathrm{m}$ at the intensity $1 \times 10^{10}$ electrons/bunch[8]. Each BPM uses four strip-lines embedded in the gaps of a quadrupole magnet. Thus the BPM measures the beam position in the middle of the quadrupole, which provides an ideal situation for the beambased alignment. Each strip-line has an independent circuit with an rf booster ampli®er before the low-pass ®lter to reduce the noise level. The calibration of the BPM is done after installation in the quadrupole, at the same time as the measurement of the magnetic center of the quadrupole using a common single wire. The actual resolution of the BPM was veri®ed by the beam using the lattice-diagnostics technique described later, and the result was $\sigma=1.6 \mu \mathrm{m}$ at $N=0.7 \times 10^{10}$, which is just equal to the design number.

\section{G. Cavity beam position monitor}

The detection of the beam position at the FP is extremely useful in the diagnostics of FFTB. It has many usages: to measure and adjust the $R_{34}$ components of the transfer matrix from a well-established pre-focus to the FP to adjust the waist at the FP; to measure the vibration of the position at the FP to distinguish the position jitter and the spot size in the spot-size monitor; to measure the correlation of the beam-jitter at the FP with upstream or downstream BPMs to analyze the source of the jitter, etc. Therefore a BPM with nanometer pulse-to-pulse resolution is required. The recently developed cavity BPM[9] which detects the excitation of the $T M_{110}$ mode of a single-cell chokemode cavity at C-band $(5712 \mathrm{MHz})$ has such resolution of $1 \mathrm{~nm}$. This BPM will be fully commissioned in the next run of FFTB.

\section{H. Wire scanners}

The wire scanners are the essential tool to measure the beam pro®le at all locations in FFTB. Even at the FP it is a very powerful tool to adjust the waist in the range $\sigma_{y} \geq 0.5 \mu \mathrm{m}$. Although the wire scanners of FFTB are basically inherited from SLC[10], several new arrangements were done. One of them is a set of wires with very small tilt angles in the $x-y$ plane. A scanner with a yoke of three wires with angles $-0.7^{\circ}, 0^{\circ},+0.7^{\circ}$ is installed in one of the pre-focus points where the aspect ratio of the beam is 1:400. This scanner successfully reconstructed the beam sizes in $x, y$ and the tip angle from three apparent spot sizes. 


\section{Laser-Compton spot-size monitor}

The Laser-Compton spot-size monitor[11] measures the modulation of the Compton-scattering rate across an interference fringe of two laser lights produced at the FP. The depth of the modulation corresponds to the spot size at FP. The fringe is produced by a pulsed YAG-laser with $1.06 \mu \mathrm{m}$ wavelength, $10 \mathrm{~ns}$ pulse length, $200 \mathrm{~mJ} /$ pulse, and $10 \mathrm{pps}$ repetition rate. Since the pitch of the fringe pattern is so rigidly determined by the wavelength of the laser, the measurement of the size by this monitor is direct. This monitor can produce three different fringe patterns with different measuring ranges: $0.8 \mu \mathrm{m} \leq \sigma_{x}^{*} \leq 4 \mu \mathrm{m}, 150 \mathrm{~nm}$ $\leq \sigma_{y}^{*} \leq 750 \mathrm{~nm}$, and $40 \mathrm{~nm} \leq \sigma_{y}^{*} \leq 200 \mathrm{~nm}$.

\section{J. Ion-Scattering beam-size monitor}

The Ion-Scattering beam-size monitor[14] measures the interaction between the electron beam and ions created from the gas injected at the FP. It consists of eight multi channel plates surrounding the FP through a narrow slit which is necessary to reject the ions outside the focal length of $\beta_{y}^{*}=100 \mu \mathrm{m}$. This monitor measures two quantities: the time-of-ight and the azimuthal distribution of the scattered ions. The former tells the larger dimension, and the latter the aspect ratio and the tilt angle of the beam-spot at the FP. This monitor has an advantage in its very wide dynamic range and the insensitivity to the position jitter. Another aspect of this monitor is that the angular distribution involves more information on the beam pro®le than its sizes such as the beam-tail produced by the wake ®eld.

\section{BEAM TUNING TECHNIQUES}

\section{A. Beam-based alignment}

In order to optimize the tunability and reduce the background for the monitors in FFTB, it is necessary to align strong quadrupole and sextupole magnets with beam-based measurements[12]. Quadrupoles are aligned using a shunt technique, with resolutions from $50 \mu \mathrm{m}$ down to $0.7 \mu \mathrm{m}$. Sextupoles are aligned by moving transversely, looking at the kicks of the orbit observed in the downstream BPMs. The resolution of the residual misalignment of sextupoles by this method was 5 to $20 \mu \mathrm{m}$. All magnets were then moved into aligned positions via the magnet movers with the sub-micron resolution. The beambased alignment was done in the beginning of every successive run of FFTB.

\section{B. Lattice diagnostics}

The amount of the strength error of focusing elements in the FFTB optics must be within a few times $0.1 \%$, otherwise the machine looses the tunability to the design spot size. Thus the detection of the strength error is an important subject prior to the tuning of the machine. For this purpose, a lattice diagnostics with bump orbits is applied. A locally closed bump orbit is created by combining three kicks at quadrupoles, generated by the magnet movers. Then the orbit data from the BPMs up/down stream of the bump orbit contains the information of the strength error of the quadrupoles used in the bump, as well as the calibration errors of BPMs and movers, etc. To separate the strength error from other variables, orbits with multiple bumps are taken and multi-variable ${ }^{\circledR} \mathrm{t}$ is applied. This analysis was able to demonstrate that the FFTB quadrupole strengths were uniform to the required level of $0.1 \%$.

\section{Incoming beta match}

Because of the limited space in the beam line, the measurement and matching of the incoming phase space is done in the matching section at the entrance of FFTB which has $® v e$ quadrupoles and two skew quadrupoles[13]. The measurement is done by varying quadrupoles against the beam sizes measured by a wire scanner with three wires $x, y$, and tilted $u$. It is possible to determine all 10 parameters of the incoming beam with this method. The matching is done for $\alpha_{x, y}, \beta_{x, y}$, and two of $x-y$ coupling coef $\AA$ cients, and the remaining two $x-y$ coupling terms are less relevant to the $®$ nal spot due to the ${ }^{-}$atness of the emittances and the spot size. The matching is con®rmed at two prefoci (one for each plane) in the middle of the chromaticity correction sections with the wire scanners. This con®rmation was extremely important to tell whether the source of the error in the (n)al spot is, in the incoming beam or in the optics between the pre-foci and the FP.

\section{Tuning by multi knobs}

After the beam-based alignment, lattice diagnostics, and beta match, the tuning of the spot size is done by scanning several knobs looking at the spot size measured by the spot-size monitors[13], [2]. Since these monitors are located at different focal points, the tuning must be done separately for each monitor. The tuning at one monitor, however, signi ${ }^{\circledR}$ cantly reduces the number of steps of tuning for another monitor. Usually the tuning is done with a beam with reduced momentum spread, $\Delta p / p \leq 0.1 \%$ to avoid the mixing of linear and higher order effects. The knobs for the linear terms, such as the waist position, dispersion, and one of the $x-y$ coupling $\left(x^{\prime} y^{\prime}\right)$ are always effective to reduce the spot, but its magnitude depends on the condition. Several iterations are necessary to reach the minimum. These knobs are built by either changing the strength of magnets or combination of the sextupole movers.

FFTB has four strong sextupoles for the chromaticity correction. Each sextupole has the high precision cam-shaft mover. Knobs for the waist motion and linear dispersions in $x$ and $y$, one of the $x$ - $y$ coupling ( $x^{\prime} y^{\prime}$ term) can be made by particular combinations of these sextupole movers. According to the high precision, the repeatability, and the hysteresis-free nature of the cammover, these mover knobs have advantages on a small-range, repeated scan of the knobs. These knobs were frequently used in the tuning, and after the value of the knob is optimized, their motions were replaced with equivalent changes of the magnet strengths of dipoles, quadrupoles, and skew quadrupoles.

Another effective set of knobs was the nonlinear multi knobs which control four sextupole terms $\left(x^{\prime 3}, x^{\prime} y^{2}, x^{\prime 2} y^{\prime}, y^{\prime 3}\right)$ independently by combining two normal and two skew trim sextupoles.

\section{OBSERVATION OF NANOMETER SPOT}

FFTB has run for about seven weeks in four periods since September 1993. The ®rst fringe pattern of the Laser-Compton 

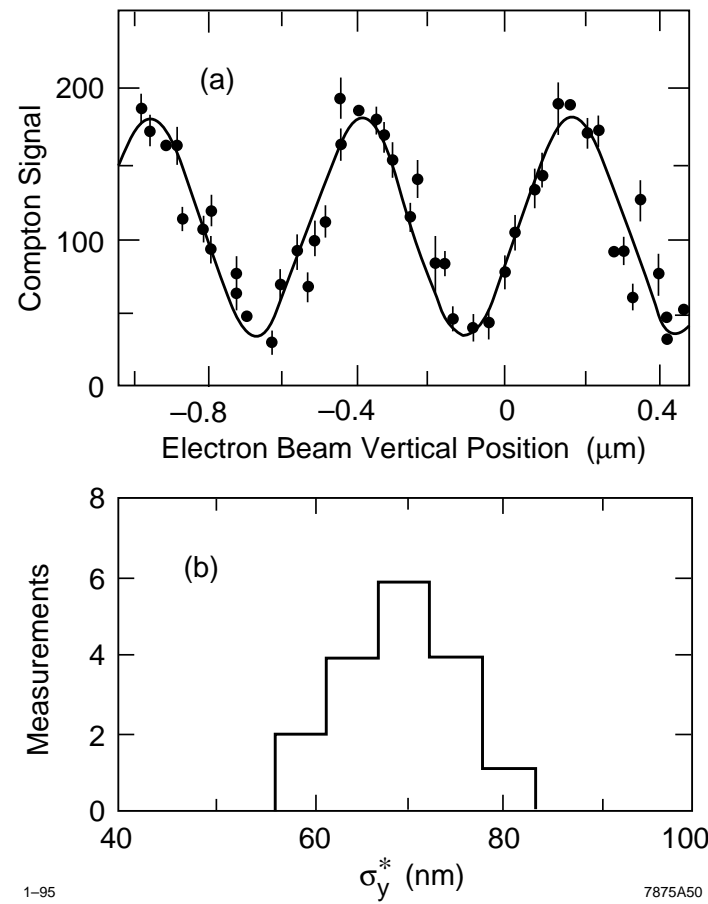

Figure. 2. (a) Typical vertical fringe pattern seen by the LaserCompton monitor. This example corresponds to $\sigma_{y}^{*}=70 \mathrm{~nm}$. (b) The distribution of the measured in the last 3 hours of the run in May 1994.

monitor was observed in the beginning of April 1994 in horizontal. The small vertical spot around $70 \mathrm{~nm}$ was observed in the middle of May 1994[15], [11]. Figure 2 shows the typical fringe pattern seen by the monitor, and also the distribution of the measured spot sizes during the last 3 hours of the run in May 1994. This distribution shows that the beam line of FFTB has the stability of $\sigma_{y}^{*}=70 \pm 7 \mathrm{~nm}$ including the possible ${ }^{-}$uctuations in the monitor.

The Ion-Scattering monitor was commissioned partly in May 1994, and more totally in September 1994[15], [14]. The spot size determined from the time-of--ight and the azimuthal distribution was typically $1.6 \mu \times 80 \mathrm{~nm}$, which agrees well with the results of the Laser-Compton monitor. Figure 3 shows a typical azimuthal distribution of the scattered ions observed by the monitor. Taller peaks indicate the higher aspect ratio, i.e., smaller vertical size.

The spot sizes above are obtained with a con $\AA_{\text {guration }} \beta_{x}^{*}=$ $10 \mathrm{~mm}$ and $\beta_{y}^{*}=100 \mu \mathrm{m}$ with the incoming emittances around $\gamma \varepsilon_{x}=30 \mu \mathrm{m}, \gamma \varepsilon_{y}=1 \mu \mathrm{m}$ with the momentum spread $\Delta p / p=$ $\pm 0.1 \%$. The horizontal focusing was relaxed to reduce the background signal of the monitor. Although the observed spot sizes were already close to the design number, it was also true that there were non-negligible discrepancies in the vertical sizes between the measured and the expected from the emittance and $\beta_{y}^{*}$. The discrepancy appeared similar in both spot-size monitors. Figure 4 shows the squared vertical spot size measured by the Laser-Compton monitor versus the incoming vertical emittance. The linear $® t$ Fit \pm 1 in Fig. 4 may suggest an existence of an error source which give an offset to the measured spot size, such as a vibration of the ${ }^{\circledR}$ nal lenses. The ${ }^{\circledR} t$ with the restriction

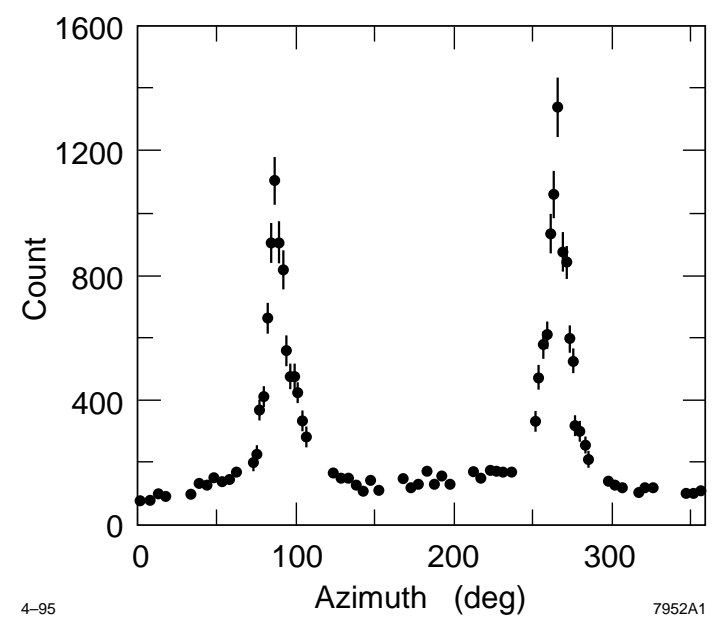

Figure. 3. Azimuthal distribution of scattered He ion detected by the Ion-Scattering monitor in September 1994. This distribution corresponds to $1.6 \mu \mathrm{m} \times 80 \mathrm{~nm}$ spot.

at the origin (Fit \pm 2$)$, however, is also possible with the magnitude of the errors. Further experiment such as a measurement of the vibration jitter of the beam using the cavity BPM is necessary to complete our investigation of the results of the spot size.

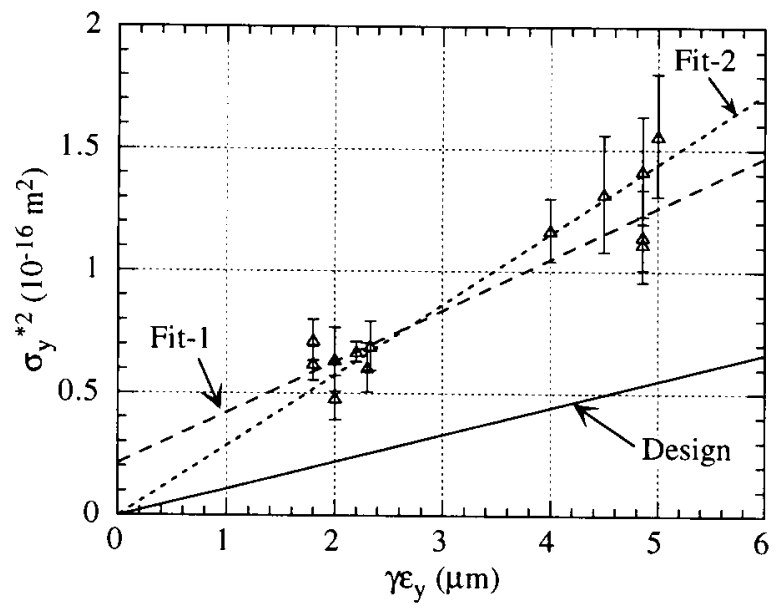

Figure. 4. Vertical spot size (squared) versus the incoming vertical emittance. Measured in September 1994 with the LaserCompton monitor. Fit \pm 1 (dashed) is the linear $® t$ to the measured data, Fit \pm 2 (dotted) is the linear $® t$ passing the origin, and the solid is the design line.

\section{CHROMATIC BEHAVIORS}

The chromatic behavior of the FFTB optics is measured in several ways. First we looked at the shift of the vertical waist position at the FP by changing the incoming beam energy. The energy spread of the beam is set small, $\Delta p / p \leq \pm 0.1 \%$. If the chromaticity correction works properly as designed, the shift of the waist is minimized within the design bandwidth. Its behavior is shown in Fig. 5 as the solid curve. The shift of the vertical waist position was measured by waist scans with the LaserCompton monitor in September 1994. First the results shown as squares in Fig. 5 were obtained, which signi ${ }^{\circledR}$ cantly deviate 
from the design curve. At that time the lattice diagnostics with multi-bump had been performed and predicted the common calibration error of the quadrupoles relative to the beam energy (to the main dipoles) by $-0.73 \%$, i.e., the beam had higher energy offset of $+0.73 \%$. The expected shift of the waist with the predicted the energy offset becomes the dashed line of Fig 5, which now well agrees with the measured (squares). Then we corrected the energy offset, and re-measured the waist position as shown by crosses in Fig. 5. The results became closer to the design, but still deviated from the design by a small amount. At that time it was found that the vertical sextupoles had been trimmed by about 3\% stronger than the design. The trimmed sextupoles give the expected curve as the dot-dashed line in Fig. 5, which agrees with the measurement (crosses) quite well.

The second veri®cation of the chromatic behavior was the measurement of the spot size by changing the momentum spread of the incoming beam through varying the phase-ramp of the Linac. We changed the momentum spread from $\Delta p / p= \pm 0.1 \%$ to $\pm 0.5 \%$, and the measured vertical spot size at the FP stayed almost constant within the measurement error of about $10 \%$. This shows that the chromaticity correction works, since the bare chromaticity is so large as shown in Fig. 1.

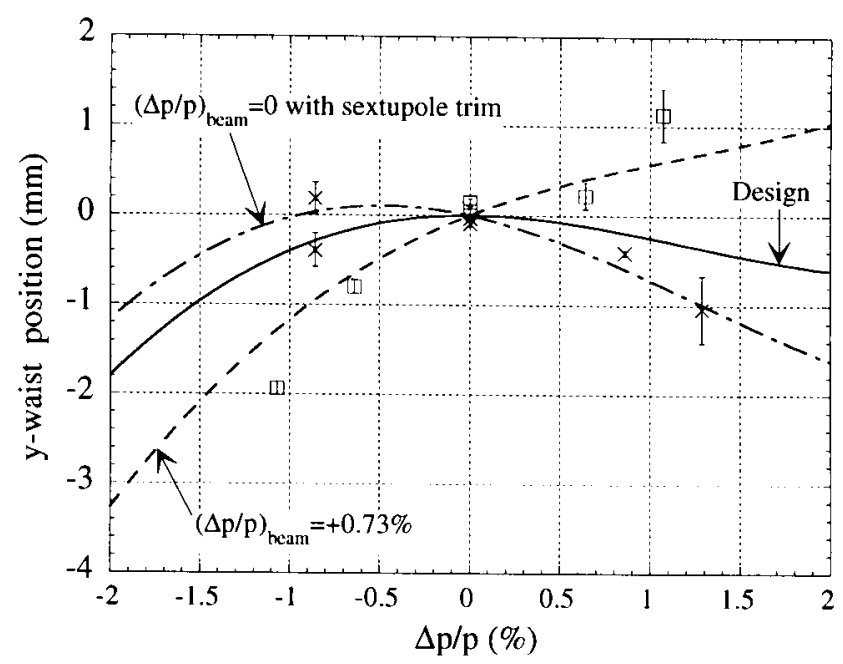

Figure. 5. Shift of the vertical waist position as a function of the momentum offset of the incoming beam, measured at the LaserCompton monitor. Squares are measured before the correction of the beam-energy offset. Dashed line is expected from the energy offset of $+0.73 \%$. The crosses are measured after the correction. The dot-dashed is expected from the trimmed values then applied to the sextupoles. The design is shown by the solid line.

\section{References}

[1] M. Berndt et al., Final Focus Test Beam Design Report,SLAC $\pm \mathrm{REF} \pm 376(1991)$.

[2] K. Oide, SLAC \pm PUB \pm 4953 , in Proceedings of PAC89, Chicago(1989);J. Irwin et al., SLAC $\pm \mathrm{PUB} \pm 5539$, in Proceedings of PAC91, San Fransisco(1991);G. Roy, SLAC \pm REF \pm 397 , Ph.D. thesis(1992).

[3] H. Nakayama et al., FFTB $\pm 94 \pm 4(1994)$.

[4] F. Bulos et al., SLAC \pm PUB \pm 5485 , in Proceedings of PAC91, San Fransisco(1991).
[5] N. Ishihara et al., KEK \pm Preprint $\pm 92 \pm 89$, in Procedings of HEAC92, Humburg(1992).

[6] V. E. Bressler et al., SLAC \pm PUB \pm 6152 , in Proceedings of PAC93, Washington DC(1993).

[7] G. Bowden et al., SLAC \pm PUB $\pm 6132(1993)$;G. Heimlinger, $\mathrm{MPI} \pm \mathrm{PhE} 93 \pm 13$, Ph.D. thesis(1993).

[8] H. Hayano et al., Nucl. Instrum. Meth. Phys. Res., A320(1992)47.

[9] S. Hartman et al., in these proceedings.

[10] R. C. Field et al., Nucl. Instrum. Meth. Phys. Res., A295(1990)279.

[11] T. Shintake, Nucl. Instrum. Meth. Phys. Res., A311(1992)453; T. Shintake et al., KEK \pm Preprint $\pm 92 \pm 65$, in Proceedings of HEAC92, Hamburg(1992);T. Shintake et al., KEK \pm Preprint $\pm 94 \pm 129$ (1994).

[12] P. Tenenbaum et al., in these proceedings;F. Bulos et al., SLAC \pm PUB \pm 5485 , in Proceedings of PAC91, San Fran$\operatorname{sisco}(1991)$.

[13] P. Tenenbaum et al., in these proceedings.

[14] J. Buon et al., Nucl. Instrum. Meth. Phys. Res., A306(1991)93;P. Puzo et al., LAL \pm RT $\pm 94 \pm 08$, in Proeedings of EPAC94, London(1994).

[15] V. Balakin et al., Phys. Rev. Lett. 74(1995) 2479. 